\title{
Transcranial Direct Current Stimulation Combining Mindfulness-Based Relapse Prevention for Smoking Cessation: A Case Report
}

\author{
Zahra Khayyer $^{1 *(\infty)}$, Razieh Saberi Azad $^{2}{ }^{\circledR},{\text { Mohsen } \operatorname{Dadjoo}^{3}}^{\circledR}$, Azam Shojaei $^{(\oplus)}$ \\ 'Educational Sciences \& Psychology Department, University of Isfahan, and Lecturer in Almahdi Mehr Isfahan Institute of \\ Higher Education, Isfahan, Iran \\ ${ }^{2}$ Human Sciences Department, Sepahan Institute of Higher Education, Isfahan, Iran \\ ${ }^{3}$ Institute for Cognitive and Brain Sciences, Shshid Beheshti University, Tehran, Iran \\ ${ }^{4}$ Educational Sciences \& Psychology Department, Azarbaijan Shahid Madani University, Tabriz, Iran
}

\begin{abstract}
Previous electroencephalography (EEG) researches have shown significant differences in terms of abnormal brain activity among smokers and non-smokers. Typically, EEG analysis showed decreased slow waves and increased fast waves in people with nicotine consumption. The present study tested a transcranial direct current stimulation (tDCS) technique combined with Mindfulness-based relapse prevention (MBRP) to reduce nicotine cravings. Specifically, cathodal tDCS over left prefrontal cortex (PFC), as well as anodal stimulation over the right central zone, tested. An 18-year-old right-handed male with almost 3 years of smoking participated. He had no anti-tobacco medical prescription. The participant received twelve sessions of tDCS treatment (2 times a week). Anodal stimulation (1.5 $\mathrm{mA}, 15$ minutes) performed on C3 (LORETA source localization) and cathodal stimulation on FP2. Also, the participant received 30-minute MBRP-based psychotherapy sessions in order to ameliorate aggression, stress, and craving. Clinical evaluations, Buss-Perry Aggression Questionnaire (AGQ), Fagerstrom Test for Nicotine Dependence (FTND), Perceived Stress Scale (Cohen, Kamarck, \& Mermelstein, 1994), and daily checklist for the number of smoked cigarettes administered along with carbon monoxide (CO) levels. Also, subjective measures of craving intensity between zero and ten measured after treatment and 3-month follow-up. After treatment, the participant showed a substantial reduction in all clinical tests, and the number of cigarettes decreased from 30 to 2 a day. From a psycho neurotherapeutic perspective, the present study provides preliminary indications for a novel and efficient treatment of smoking during adolescence. Further studies are needed to determine generalizability and overall efficiency.

Keywords: Cigarette smoking, transcranial direct current stimulation (tDCS), quantitative electroencephalogram (QEEG), mindfulness-based relapse prevention (MBRP).
\end{abstract}

*Correspondence to Zahra Khayyer, PhD in Psychology, University of Isfahan, Azadi square, Isfahan, Iran.

Tel: +989380171218,

Email: z.khayer62@yahoo.com

Published online August 31, 2019

Citation: Khayyer Z, Saberi Azad R, Dadjoo M, Shojaei A. Transcranial direct current stimulation combining mindfulness-based relapse prevention for smoking cessation: a case report. Int Clin Neurosci J. 2019;6(3):118-122. doi:10.15171/icnj.2019.22.

\section{Introduction}

Human society is plagued by tobacco use more than ever. One person dies every 10 seconds due to diseases caused by or associated with tobacco use. ${ }^{1}$ World Health Organization estimated that smoking cigarette causes four million deaths a year in the world, and estimates 10 million deaths a year until 2030. Adolescents are particularly vulnerable to this problem. Adolescence is a period with broad developments in cognitive, biological, and psychological domains. Many adolescents are involved in high-risk behaviors such as consuming alcohol, tobacco, or illegal substances. These high levels of tobacco and alcohol consumption among adolescents are related to severe health problems such as cardiovascular diseases and cancer. ${ }^{2}$ In Iran, 2.5 to $17 \%$ of male adolescents smoke cigarettes; tobacco consumption is a prevalent problem. In Europe, the problem has even more pronounced because about $22 \%$ of male adolescents smoke. ${ }^{3}$

Nicotine craving is a central aspect of smoking addiction. Research findings have shown that craving plays an integral part in post-treatment relapses and continued consumption. Craving is an uncontrollable desire for consumption. This desire, if not satisfied, can cause psychological and physical problems such as fatigue, anorexia, anxiety, insomnia, aggression, depression, and attention problems. Unsurprisingly, craving is strongly associated with relapse as people who overcome their initial substance use have a $70 \%$ relapse rate reported a

(C) 2019 The Author(s). This is an open access article distributed under the terms of the Creative Commons Attribution License (http:// creativecommons.org/licenses/by/4.0/), which permits unrestricted use, distribution, and reproduction in any medium, provided the original work is properly cited. 
year later. ${ }^{4}$

The rewarding experience of drugs use is associated with a complex neural circuit in the cortico-subcortical network. This circuit includes the striatum, insula, hippocampus, amygdala and the orbital prefrontal cortex (PFC). ${ }^{5}$ This network and a mesolimbic pathway, through the neurotransmitter dopamine, probably play an essential part in understanding drug craving and relapse. For instance, magnetic resonance imaging (MRI) studies have detected reduced gray matter integrity among smokers in several brain regions including the $\mathrm{PFC}$, anterior cingulate cortex (ACC), insula, thalamus, and cerebellum. ${ }^{6}$

Also, some information about the brain and addiction come from electroencephalography (EEG) studies. For instance, a study has found that slow frequencies are associated with the activity of the dopaminergic brain reward system in which smokers have decreased delta power while resting. Furthermore, increased synchrony in beta frequencies during temporary heroin withdrawal may increase reward-driven behavior. Also, higher resting beta power among alcoholics can interpret as cortical hyperexcitability. ${ }^{7}$ In a similar vein, qEEG studies have shown connections between beta-band activity and failure to stay away from drugs. Thus far, research suggests that nicotine deprivation among smokers is associated with the lesser power in beta bands. In general, nicotine appears to reduce slower wave power and increase faster wave power. ${ }^{8}$

One perspective on addiction is to treat it with psychotherapeutic methods such as mindfulness. It is a method of cultivating a way of being in the world that was first practiced by the man known as Gautama, and later Buddha. The mindfulness practitioner tries to stay in the present moment by focusing their attention on breathing or bodily experiences. Scientists developed the Mindfulness-based relapse prevention (MBRP) program for the treatment of addictive behavior. The MBRP based on the practitioner's ability to detect triggering stimuli, habits, and immediate responses to connect inner mental states with outside stimuli-driven experiences. By learning to create new responses and emotions from outside stimuli, the MBRP aims to make patients less susceptible to high-risk situations. There are some indications that the reactivity of the emotional brain systems between the frontal executive and the limbic system changed by mindfulness. ${ }^{9}$ A key feature of mindfulness practice is the reduction of avoidance and fear, which has been shown to ameliorate stress. ${ }^{10}$ As addictive behavior accompanies hyperactivity of the limbic system, mindfulness-based training may help to calm hyperactivity.

An alternative treatment method to help smoking cessation is transcranial direct current stimulation (tDCS). The tDCS is a non-invasive focal brain stimulation in which a weak, direct current (1-2 mA) is conducted through the scalp using a pair of rubber carbon pads $\left(10.5 \mathrm{~cm}^{2}\right)$. Direct stimulation of the brain can affect two features that are dysfunctional in tobacco-dependent participants; namely, disinhibition which is associated with the $\gamma$-aminobutyric acid (GABA) system, ${ }^{11}$ and experience of reward and motivation which connected to the dopamine system. ${ }^{12}$

Risk-aversion, which is associated with activity in the dorsolateral prefrontal cortex (DLPFC), is less pronounced among smokers compared to non-smokers. ${ }^{13}$ One study found that tDCS over the DLPFC reduced nicotinebased cigarette consumption. Also, bilateral cathodal stimulation of the frontal-parietal-temporal association area reduced cue-related attention and smoking behavior. ${ }^{14}$ Furthermore, tDCS applied over DLPFC reduced alcohol craving. Repeated usage of tetrahydrocannabinol (THC) has been associated with increased alpha band hypercoherence between the brain's hemispheres and changed phase synchrony. Also, researchers found that smoking cue exposure increased prefrontal connectivity (which is related to cognitive control).

Nicotine dependency has complex psychopathology, including comorbidity of different psychiatric disorders, and paying attention to individual factors should be prioritized in smoking cessation treatment. Most interventions apply only one approach. ${ }^{15}$ The present study aims to measure the efficiency of MBRP in combination with tDCS in an adolescent suffering from nicotine addiction, aggressive behavior, and stress. This case study is the first of its kind to explore both behavioral and neurological modification to treat nicotine addiction.

\section{Case Report}

The participant was an unemployed 18-year-old righthanded male who had been a smoker for almost three years. He had a girlfriend, and he played the guitar very proficiently. His mother complained that he was spending too much time with his friends instead of studying, and he was falling behind in school without clear plans or dreams. His nicotine consumption consisted of more than 31 cigarettes a day. He had tried marijuana once, and he drank a moderate amount of alcohol for recreational use. He was admitted to a psychotherapy and counseling center citing nicotine addiction. He consented to the treatment and agreed to adhere to the treatment protocol (there was no record of prior use of medications). qEEG measured before and after the end of twelve sessions of interventions (tDCS plus MBRP). Nineteen active and two references EEG channels were recorded using Ag/ $\mathrm{AgCl}$ electrodes in a linked-ear montage. In order to minimize aliasing effects, a band-pass filter between 0.1 to $35 \mathrm{~Hz}$ in 250 samples per minute used. A 10-20 electrode arrangement used, and electrodes impedance kept under five $\mathrm{k} \Omega$. The EEG recordings were conducted in both open and closed eye conditions for five minutes, respectively. Ninety-second intervals of artifact-free signals used, and all recordings visually inspected. EEG signal processing data processed with MATLAB 7.11.0 software and EEG 
coherence of 5 frequency bands. In total, 171 electrode pairs $x 5$ bands in 5 bandwidths were measured; $1-4 \mathrm{~Hz}$ (delta), 4.5-7 Hz (theta), 7.5-12 Hz (alpha), 12.5-24.5 Hz (beta), and $25-30 \mathrm{~Hz}$ (high beta).

tDCS delivered by a tapwater soaked sponge pair of rubber carbon pads $\left(10.5 \mathrm{~cm}^{2}\right)$ fixed by rubber bands on the head. Through 12 sessions of treatment, the patient first received tDCS then MBRP for 2 times a week (a $2 \times 1$ design with sham and active TDCS paired with MBRP). The subject gave him written informed consent and compensated for participation. The subject informed that this is not an established first-line therapeutic option for smoking cessation. The information sheet comprised the explanation of various adverse effects of tDCS such as itching and tingling skin sensation, skin reddening, and headache. There was no information on the differences between active and sham condition.

In MBRP time, he participated in 60-minute psychoeducational sessions. During tDCS, anodal stimulation (1.5 mA, 15 minutes) performed on C3 based on LORETA source localization and cathodal stimulation on FP2. The sham tDCS mode starts with a variable ramp-in and ramp-out phase, followed by an impedance control mode with small measuring pulses of $100 \mathrm{e} 200$ $\mathrm{mA}$ amplitude every $400 \mathrm{e} 550 \mathrm{~ms}$ for the same period as in the active condition, and ends with another ramp-in and ramp-out phase. The tDCS was passive without any engagement in an online task. Using the qEEG to guide the treatment sites is recommended for making the treatment more precision based in this case, C3 instead of F3 used. Both anodal and cathodal stimulation delivered by an electrical stimulator device (Active dose II). Safety guidelines specified by Nitsche used.

To measure behavioral outcomes, the Buss-Perry Aggression Questionnaire (AGQ), Fagerstrom Test for Nicotine Dependence (FTND), and Perceived Stress Scale, before and after treatment, and 3-month follow-up administered. Craving measured on a 10-point scale, and the cigarette consumption was self-reported during the treatment sessions. Carbon monoxide (CO) levels were taken as well to validate self-reported smoking abstinence biochemically. The standard indication of tobacco use has been CO levels above 8 to $10 \mathrm{ppm}$.

\section{Results}

The participant reported reduced levels of aggression, stress, and nicotine dependence according to the clinical assessments which had maintained at 3-month follow-up (see Table 1). Analysis of the neurological data (qEEG) yielded elevated theta and decreased beta and alpha bands (see Figure 1). Analysis of self-reported craving and cigarette consumption revealed dramatic improvement in both measures (see Figure 2). Participant's CO measure taken during the first treatment session compared to his CO measures taken during Weeks 2 to 6 (see Figure 3). It explained to him that this decrease was representative of
Table 1. Clinical Assessment

\begin{tabular}{lccc}
\hline Measures & Pre-test & Post-test & 3-month follow-up \\
\hline Nicotine Dependence & 8 & 1 & 2 \\
Aggression & 98 & 87 & 79 \\
Stress & 23 & 7 & 5 \\
\hline
\end{tabular}

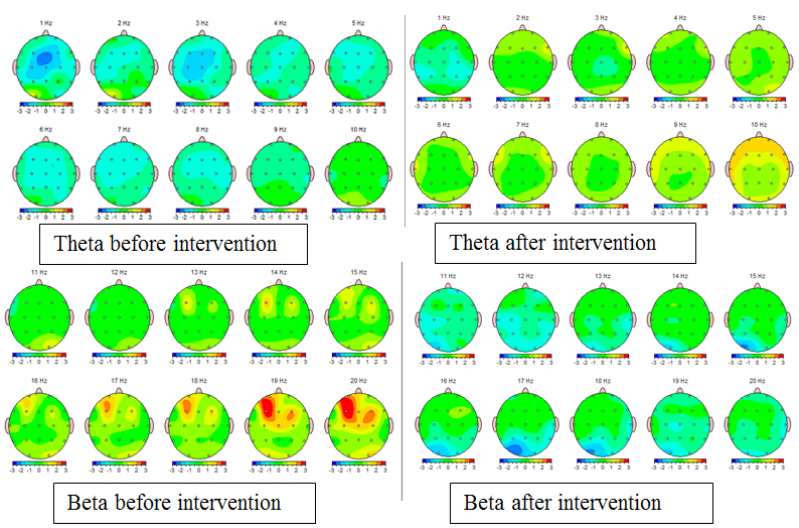

Figure 1. qEEG (Theta and Beta Bands) Before and After the Intervention.

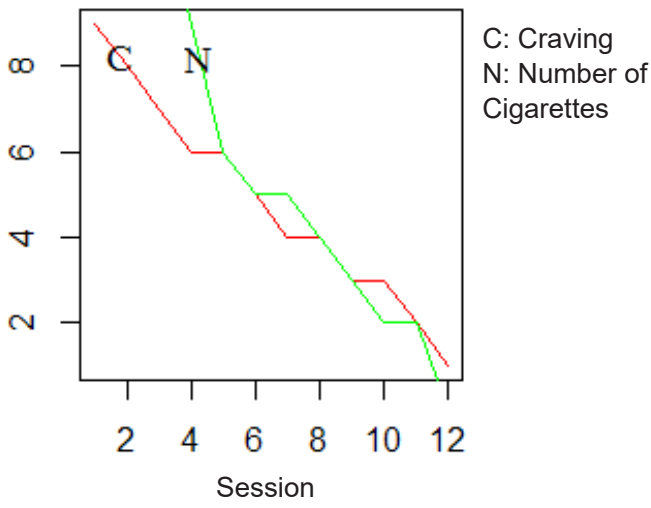

Figure 2. Changes in Craving and Cigarette Consumption After Treatment.

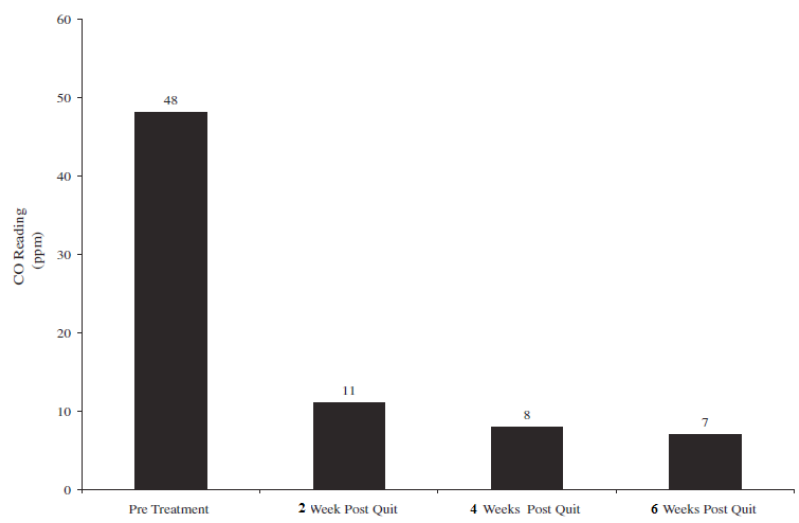

Figure 3. Participant's CO Readings During Treatment Sessions. 
the CO levels in his blood dropping and the oxygen levels in his blood increasing.

\section{Discussion}

The present study aimed to measure the efficiency of combining qEEG-based tDCS and mindfulness therapy in an 18-year old male with nicotine addiction. The results were promising, as the participant showed improvements in both neurological and clinical measurements. The results indicated an initial reduction in brain over-activity, particularly in the Beta band. Previous tDCS studies support the usefulness of electrical stimulation to improve craving and inhibition control among smokers. ${ }^{13,14}$ Given this pattern of results, reduced neural hyperactivity may lead to decreased nicotine craving. Furthermore, the participant showed more normal theta band power after receiving the intervention. A possible explanation is that the participant improved his mindfulness skills, which in turn reduced impulsivity and theta activity. Breathing and meditation techniques have been shown to stimulate theta brain wave activity. These techniques may help the brain to relax and reduce feelings of anxiety and improve emotional control, which may last for couples of months. ${ }^{16}$

Studies have shown that the application of tDCS in prefrontal areas changed decision-making strategies. One common denominator between decision-making and addictive behavior is impulsivity. Decreased impulsivity could lead to reduced cravings indirectly. ${ }^{17}$ Mindfulness techniques (see Table 2) appear to be sufficiently powerful to minimize the neural features of craving. Also, previous studies found that mindfulness reduced connectivity between the subgenual AAC and other craving-associated brain regions. ${ }^{18}$ Thus, both tDCS and mindfulness have the potential to change the neural basis for nicotine addiction. Also, changes in attention control, emotional regulation, and self-awareness may improve by mindfulness training.

The results also showed decreased cigarettes consumption. This is consistent with previous tDCS studies that showed reduced self-reported smoking. Furthermore, stress and aggression levels reduced. Mindfulness is one

Table 2. MBRP Program

\begin{tabular}{ll}
\hline Session & Techniques \\
\hline 1 & Introducing autopilot \& strategies of the restless mind \\
2 & Urge surfing \\
3 & Raisin meditation \\
4 & Mindful breathing \\
5 & Body scan \\
6 & Self-compassion meditation \\
7 & Mindful listening \\
8 & Knowing smoking triggers \\
9 & Imaginal exposure to cigarette use triggers \\
10 & Walking mindfulness \\
11 & $\begin{array}{l}\text { STIC technique (Stop, Take a breath, Imagine the future } \\
\text { consequences, and Choose) }\end{array}$ \\
12 & Reviewing and completing post-tests \\
\hline
\end{tabular}

technique to reduce stress and anxiety. This increases physical and psychological wellbeing by improving mood and satisfaction while reducing aggressiveness. Mindfulness can reduce aggression through declining rumination and subsequent physiological arousal. ${ }^{10}$

Additionally, our results revealed hyperactivity in the left motor cortex before treatment cigarette addiction. Activity in left hemisphere dorsal motor cortex is probably caused by right-handed smoking practice, assuming that the right-handed participant regularly used his right hand for nicotine intake. This is related to stimulating C3, behind the sulcus in the sensory area, which represents sensorimotor integration when applying tDCS. ${ }^{19}$ And the participant showed gradual inhibition of qEEG overactivation in the right orbitofrontal cortex. Moreover, in previous tDCS studies, a protocol-based location of stimulation is more frequent than the qEEG-based location of stimulation. For instance, researchers ${ }^{20}$ used locations of stimulation based on protocols rather than more specific and sensitive qEEG measurements used in the present study.

In conclusion, the present study shows a promising strategy to ameliorate smoking cessation. Application of qEEG-based tDCS and MBRP improved both neurological and behavioral measures. Presumably, comprehensive treatments for addictive behaviors are necessary because of the compulsive nature of dependency. Although the present study lacks a sufficient number of participants for general conclusions, the results are so promising that the study warrants further research.

\section{Conflict of Interest Disclosures}

The authors declare that they have no conflict of interests.

\section{Ethical Statement}

Consent form was completed by participant before starting the therapy. He informed that he can withdraw his consent at any time without prejudice.

\section{Acknowledgment}

We want to express our gratitude to all those who supported conducting this research project and helped us significantly improve the quality of the manuscript.

\section{References}

1. Kazem M, Noorbala A, Majdzadeh R, Karimlo M. The changing process of smoking prevalence in Iran (1991-1999): based on two national projects of health and disease. Hakim Journal. 2000;197:290-4. [Persian].

2. Scholte RH, Poelen EA, Willemsen G, Boomsma DI, Engels RC. Relative risks of adolescent and young adult alcohol use: the role of drinking fathers, mothers, siblings, and friends. Addict Behav. 2008;33(1):1-14. doi: 10.1016/j.addbeh.2007.04.015.

3. Baska T, Warren CW, Basková M, Jones NR. Prevalence of youth cigarette smoking and selected social factors in 25 European countries: findings from the Global Youth Tobacco 
Survey. Int J Public Health. 2009;54(6):439-45. doi: 10.1007/ s00038-009-0051-9.

4. Brewer J, Pbert L. Mindfulness: an emerging treatment for smoking and other addictions. J Fam Med. 2015;2(4):1035.

5. Luigjes J, van den Brink W, Feenstra M, van den Munckhof $P$, Schuurman PR, Schippers R, et al. Deep brain stimulation in addiction: a review of potential brain targets. Mol Psychiatry. 2012;17(6):572-83. doi: 10.1038/mp.2011.114.

6. Fritz HC, Wittfeld K, Schmidt CO, Domin M, Grabe HJ, Hegenscheid $\mathrm{K}$, et al. Current smoking and reduced gray matter volume-a voxel-based morphometry study. Neuropsychopharmacology. 2014;39(11):2594-600. doi: 10.1038/npp.2014.112.

7. Rass O, Ahn WY, O'Donnell BF. Resting-state EEG, impulsiveness, and personality in daily and nondaily smokers. Clin Neurophysiol. 2016;127(1):409-18. doi: 10.1016/j. clinph.2015.05.007.

8. Clarke AR, Barry RJ, Bond D, McCarthy R, Selikowitz M. Effects of stimulant medications on the EEG of children with attention-deficit/hyperactivity disorder. Psychopharmacology (Berl). 2002;164(3):277-84. doi: 10.1007/s00213-002-12050 .

9. Lutz A, Brefczynski-Lewis J, Johnstone T, Davidson RJ. Regulation of the neural circuitry of emotion by compassion meditation: effects of meditative expertise. PLoS One. 2008;3(3):e1897. doi: 10.1371/journal.pone.0001897.

10. Esmaeili M, Basiri N, Khayer Z. The effects of mindfulnessbased cognitive therapy (MBCT) on psychological well-being of mothers of children with learning disabilities. J Learn Disabil. 2016;6(20):26-39.

11. Stagg CJ, Nitsche MA. Physiological basis of transcranial direct current stimulation. Neuroscientist. 2011;17(1):37-53. doi: $10.1177 / 1073858410386614$.

12. Cosgrove KP, Wang S, Kim SJ, McGovern E, Nabulsi N, Gao $\mathrm{H}$, et al. Sex differences in the brain's dopamine signature of cigarette smoking. J Neurosci. 2014;34(50):16851-5. doi: 10.1523/jneurosci.3661-14.2014.

13. Knoch D, Gianotti LR, Baumgartner T, Fehr E. A neural marker of costly punishment behavior. Psychol Sci. 2010;21(3):33742. doi: 10.1177/0956797609360750.

14. Boggio PS, Liguori P, Sultani N, Rezende L, Fecteau S, Fregni F. Cumulative priming effects of cortical stimulation on smoking cue-induced craving. Neurosci Lett. 2009;463(1):82-6. doi: 10.1016/j.neulet.2009.07.041.

15. Khayyer Z, Ngaosuvan L, Sikstrom S, Ghaderi AH. Transcranial direct current stimulation based on quantitative electroencephalogram combining positive psychotherapy for major depression. J Integr Neurosci. 2018;17(2):89-96. doi: 10.31083/jin-170045.

16. Desai R, Tailor A, Bhatt T. Effects of yoga on brain waves and structural activation: A review. Complement Ther Clin Pract. 2015;21(2):112-8. doi: 10.1016/j.ctcp.2015.02.002.

17. Fecteau S, Fregni F, Boggio PS, Camprodon JA, PascualLeone A. Neuromodulation of decision-making in the addictive brain. Subst Use Misuse. 2010;45(11):1766-86. doi: 10.3109/10826084.2010.482434.

18. Westbrook C, Creswell JD, Tabibnia G, Julson E, Kober H, Tindle HA. Mindful attention reduces neural and self-reported cue-induced craving in smokers. Soc Cogn Affect Neurosci. 2013;8(1):73-84. doi: 10.1093/scan/nsr076.

19. Wall MB, Mentink A, Lyons G, Kowalczyk OS, Demetriou L, Newbould RD. The effect of smoking on the brain revealed by using electronic cigarettes with concurrent fMRI. bioRxiv. 2017:107771. doi: 10.1101/107771.

20. Brunoni AR, Moffa AH, Fregni F, Palm U, Padberg F, Blumberger DM, et al. Transcranial direct current stimulation for acute major depressive episodes: meta-analysis of individual patient data. Br J Psychiatry. 2016;208(6):522-31. doi: 10.1192/bjp. bp.115.164715. 